\title{
ON A THEOREM OF SCHUR
}

\author{
PETER HILTON
}

(Received 9 January 2001)

To the memory of a dear friend and colleague, Paul Olum

\begin{abstract}
We study the ramifications of Schur's theorem that, if $G$ is a group such that $G / Z G$ is finite, then $G^{\prime}$ is finite, if we restrict attention to nilpotent group. Here $Z G$ is the center of $G$, and $G^{\prime}$ is the commutator subgroup. We use localization methods and obtain relativized versions of the main theorems.
\end{abstract}

2000 Mathematics Subject Classification. 20B07, 20D15.

1. Introduction. The theorem to which we refer is that which asserts that if $G$ is a group and $Z G$ is its center, then

$$
G / Z G \text { finite } \Longrightarrow G^{\prime} \text { finite, }
$$

where $G^{\prime}$ is the commutator subgroup of $G$. This theorem has a nice homological proof, using the 5 -term exact sequence

$$
H_{2} G \stackrel{\alpha_{4}}{\longrightarrow} H_{2}(G / Z G) \stackrel{\alpha_{3}}{\longrightarrow} Z G \stackrel{\alpha_{2}}{\longrightarrow} G_{a b} \stackrel{\alpha_{1}}{\longrightarrow}(G / Z G)_{a b}
$$

derived from the short exact sequence $Z G \rightarrow G \rightarrow G / Z G$. For if $G / Z G$ is finite then $H_{2}(G / Z G)$ is finite. Thus $G^{\prime} \cap Z G=\operatorname{ker} \alpha_{2}=\operatorname{im} \alpha_{3}$ is finite. But $G^{\prime} / G^{\prime} \cap Z G \subseteq G / Z G$ is also finite, so, finally, $G^{\prime}$ is finite.

We remark that Schur's theorem has a converse which is valid if $G$ is finitely generated $(f g)$. We include a proof for completeness.

THEOrem 1.1. Let $G$ be an $f g$ group such that $G^{\prime}$ is finite. Then $G / Z G$ is finite.

Proof. Let $G=\left\langle x_{1}, x_{2}, \ldots, x_{k}\right\rangle$. Now, for any $x \in G$, there can only be finitely many distinct conjugates of $x$. For there is a one-one correspondence

$$
y^{-1} x y \longleftrightarrow x^{-1} y^{-1} x y
$$

between the set of conjugates of $x$ and a subset of $G^{\prime}$; and $G^{\prime}$ is finite. Thus $\left[G: C_{G} x\right]$ is finite for all $x \in G$, where $C_{G} S$ is the centralizer in $G$ of the subset $S$ of $G$. But if each $\left[G: C_{G} x_{i}\right], 1 \leq i \leq k$, is finite, so is $\left[G: \cap_{i} C_{G} x_{i}\right]$. On the other hand, $\cap_{i} C_{G} x_{i}=Z G$, so $[G: Z G]$ is finite. Thus, as claimed, $G / Z G$ is a finite group.

Schur's theorem, and its converse, take on a particular significance in the localization theory of nilpotent groups [1]. For it is one of the main problems in that theory to 
calculate the Mislin genus $\mathscr{G}(G)$ of an $f g$ nilpotent group $G$ and to identify its members. Here $G(G)$ is the set of isomorphism classes of $f g$ nilpotent groups $H$ such that $G$ and $H$ localize at every prime $p$ to isomorphic groups, $G_{p} \cong H_{p}$ for all primes $p$. It is shown in [2,3] that if $G^{\prime}$ is finite then $\mathscr{G}(G)$ may itself be given the structure of a (finite) abelian group, a fact which very much facilitates the study of $\mathscr{G}(G)$.

In the category of nilpotent groups (not necessarily $f g$ ) it makes sense to consider $P$-torsion groups, where $P$ is a family of primes, and to study such groups by the techniques of localization. In this way we are able to prove a $P$-torsion variant of Schur's theorem, namely,

THEOREM 1.2. Let $G$ be a nilpotent group such that $G / Z G$ is a P-group. Then $G^{\prime}$ is a P-group.

We may also prove a converse of Theorem 1.2; as with Schur's theorem itself, it is necessary to impose a supplementary finiteness condition.

THEOREM 1.3. Let $G$ be a nilpotent group such that $G^{\prime}$ is a P-group of exponent $m$. Then $G / Z G$ is a P-group of exponent dividing $m^{c-1}$, where nil $G=c$.

Actually we regard Theorems 1.2 and 1.3 as the absolute forms of our results and emphasize the relative forms which appear to be quite new. In our relativization we replace the group $G$ by a pair $(G, N)$ consisting of a nilpotent group $G$ and a normal subgroup $N$ of $G$. Then the absolute case is given by $N=G$; moreover, in our relativization, $Z G$ is replaced by $C_{G}(N)$, which is easily seen to be a normal subgroup of $G$; and $G^{\prime}$ is replaced by the commutator group $[G, N]$.

We remark that Theorem 1.2 also has a variant in which a finiteness condition is imposed just as in Theorem 1.3. Precisely, we have the following theorem.

THEOREM 1.4. Let $G$ be a nilpotent group such that $G / Z G$ is a $P$-group of exponent $m$. Then $G^{\prime}$ is a P-group of exponent dividing $m^{c-1}$, where nil $G=c$.

We will prove the relativizations of Theorems 1.2, 1.3, and 1.4 in Section 2. Proofs of the absolute forms, that is, of Theorems 1.3 and 1.4 are to be found in [4]. For Warfield proves (the case $n=1$ is the critical case).

(a) If $\Gamma_{n+1}$ has exponent $m$, then $G / Z_{n} G$ has exponent dividing $m^{c-n}$ (see [4, Corollary 2.6]); and

(b) if $G / Z_{n} G$ has exponent $m$, then $\Gamma_{n+1}$ has exponent dividing $m^{c-n}$ (see [4, Corollary 3.16]).

Here we adopt Warfield's convention that $\Gamma_{2}=G^{\prime}$ and $Z_{1}=Z G$.

We do not have available to us a homological proof of a relative version of Schur's theorem. However we do show in the appendix how we may use homological arguments to obtain Theorem 1.4 with a small loss of sharpness in our bound on the exponent of $G^{\prime}$.

A key tool in our proof of the relative version of Theorem 1.3 is a theorem on the localization of nilpotent groups due to Karl Lorensen (Theorem 2.6). This theorem is of considerable interest in its own right. It is a pleasure to acknowledge the crucial help the author received from his friend (and erstwhile student) Karl Lorensen, not only in the provision of Theorem 2.6. 
2. Localization methods. Let $P$ be a family of primes and let $Q$ be the complementary family. We first state and prove the relativization of Theorem 1.2.

THEOREM 2.1. Let $G$ be a nilpotent group and $N$ a normal subgroup such that $G / C_{G}(N)$ is a $P$-group. Then $[G, N]$ is a P-group.

Proof. Let $e: G \rightarrow G_{Q}$ localize at the family $Q$. Now $e$ maps $C_{G}(N)$ into $C_{G_{Q}}\left(N_{Q}\right)$; moreover, $C_{G_{Q}}\left(N_{Q}\right)$ is $Q$-local. Thus, in fact, the $Q$-localization $C_{G}(N)_{Q}$ of $C_{G}(N)$ must be a subgroup of $C_{G_{Q}}\left(N_{Q}\right)$, that is,

$$
C_{G}(N)_{Q} \subseteq C_{G_{Q}}\left(N_{Q}\right) \subseteq G_{Q}
$$

Now since $G / C_{G}(N)$ is a $P$-group, $\left(G / C_{G}(N)\right)_{Q}=1$, so that $G_{Q}=C_{G}(N)_{Q}$. Hence, by (2.1) $G_{Q}=C_{G_{Q}}\left(N_{Q}\right)$. Thus every element of $G_{Q}$ commutes with every element of $N_{Q}$, so that $\left[G_{Q}, N_{Q}\right]=1$. But $\left[G_{Q}, N_{Q}\right]=[G, N]_{Q}$, so $[G, N]$ is a $P$-group.

It is clear from this line of proof that, if we want a result in the opposite direction to that of Theorem 2.1, we will have to establish conditions under which

$$
C_{G_{Q}}\left(N_{Q}\right)=C_{G}(N)_{Q}
$$

Put another way, we ask when the restriction $e_{0}: C_{G}(N) \rightarrow C_{G_{Q}}\left(N_{Q}\right)$ of the $Q$ localization $e: G \rightarrow G_{Q}$ itself $Q$-localizes. Now certainly $e_{0}$ is $Q$-injective and $C_{G_{Q}}\left(N_{Q}\right)$ is $Q$-local. Thus $e_{0} Q$-localizes if and only if it is $Q$-surjective.

In seeking conditions under which $e_{0}$ is $Q$-surjective-and again in proving Lorensen's theorem (Theorem 2.6), we need to apply a basic result in [1], namely, Theorem 6.1. We quote that result here as Lemma 2.2.

LEMMA 2.2 (see [1, Theorem 6.1]). Let $G$ be a nilpotent group with nil $G=c$ and let $a, b \in G$ with $b^{m}=1$. Then $(a b)^{m c}=a^{m c}$.

However, we can, in fact, refine this result and it will be valuable to do so. Thus we may enunciate

LEMmA 2.3. If, in addition, $b \in \Gamma^{i} G$, then $(a b)^{m^{c-i+1}}=a^{m^{c-i+1}}$.

(Recall that we adopt Warfield's convention for enumerating the terms of the lower central series of $G$, so that $\Gamma^{1} G=G, \Gamma^{2} G=G^{\prime}$.)

Proof OF Lemma 2.3. We apply Lemma 2.2, but replace $G$ by $\langle a, b\rangle$. However, if $b \in \Gamma^{i} G$ then nil $\langle a, b\rangle \leq c-i+1$.

We now apply Lemma 2.2 (we will need the more refined Lemma 2.3 later) to prove the following theorem.

THEOREM 2.4. Let $G, H$ be nilpotent groups with subgroups $\bar{G} \subseteq G, \bar{H} \subseteq H$. Let $\varphi$ be a $Q$-bijective homomorphism from $G$ to $H$ sending $\bar{G}$ into $\bar{H}$, and let $\bar{\varphi}: \bar{G} \rightarrow \bar{H}$ be obtained by restricting $\varphi$. Then $\bar{\varphi}$ is $Q$-surjective (and hence $Q$-bijective) if and only if, for all $x \in G$ such that $\varphi x \in \bar{H}$, there exists a P-number $m$ such that $x^{m} \in \bar{G}$.

Proof. We for brevity, describe the property that, for all $x \in G$ such that $\varphi x \in \bar{H}$, there exists a $P$-number $m$ such that $x^{m} \in \bar{G}$ as property $S$. Suppose 
then that $\bar{\varphi}$ is $Q$-surjective, and let $x \in G$ satisfy $\varphi x \in \bar{H}$. Since $\bar{\varphi}$ is $Q$-surjective, there exists a $P$-number $n$ and an element $\bar{x} \in \bar{G}$ such that $\bar{\varphi} \bar{x}=\varphi x^{n}$. But then $x^{n}=\bar{x} z, z \in G$ with $z^{k}=1$ for some $P$-number $k$, since $\varphi$ is $Q$-injective. Let $\operatorname{nil} G=c$. Then, by Lemma 2.2, $x^{n k^{c}}=\bar{x}^{k^{c}} \in \bar{G}$ and $n k^{c}$ is a $P$-number, establishing property $S$.

Suppose, conversely, that property $S$ holds and let $y \in \bar{H}$. Since $\varphi$ is $Q$-surjective, there exists a $P$-number $n$ and $x \in G$ such that $\varphi x=y^{n}$. Thus, by property $S$, there exists a $P$-number $m$ such that $x^{m} \in \bar{G}$. Then $\bar{\varphi}\left(x^{m}\right)=y^{m n}$ and $m n$ is a $P$-number, so $\bar{\varphi}$ is $Q$-surjective.

COROLlaRY 2.5. The restriction $e_{0}: C_{G}(N) \rightarrow C_{G_{Q}}\left(N_{Q}\right) Q$-localizes if and only if, for all $x \in G$ such that ex $\in C_{G_{Q}}\left(N_{Q}\right)$, there exists a P-number $n$ such that $x^{n} \in C_{G}(N)$.

This result enables us to exploit the following theorem due to Karl Lorensen. With $G$ a nilpotent group, $N$ a normal subgroup of $G$, and $x \in G$, we write $T_{P} \Gamma_{\langle x\rangle}^{2} N$ for the $P$-primary component of the torsion subgroup of $\Gamma_{\langle x\rangle}^{2} N$, which is a subgroup of $N$ generated by commutators $\left[x^{r}, a\right], a \in N$. We then prove the following theorem.

THEOREM 2.6 (Lorensen). Let $e_{0}: C_{G}(N) \rightarrow C_{G_{Q}}\left(N_{Q}\right)$ be obtained by restricting the $Q$-localization $: G \rightarrow G_{Q}$. Then $e_{0} Q$-localizes provided that, for all $x \in G, T_{P} \Gamma_{\langle x\rangle}^{2} N$ has finite exponent.

Proof. (This is a small but significant modification of Lorensen's proof, since it exploits Lemma 2.3.) We will apply Corollary 2.5. Thus we must show that, for all $x \in G$ such that ex $\in C_{G_{Q}}\left(N_{Q}\right)$, there exists a $P$-number $n$ such that $x^{n} \in C_{G}(N)$. Now let $m=\exp T_{p} \Gamma^{2}{ }_{\langle x\rangle} N$, and let $y \in N$. Then $m$ is a $P$-number and $e[x, y]=[e x, e y]=1$, since $e x \in C_{G_{Q}}\left(N_{Q}\right)$. Hence $[x, y] \in T_{P} \Gamma^{2}\langle x\rangle N$, so $[x, y]^{m}=1$.

Now $x[x, y]=y^{-1} x y$. Hence, by Lemma 2.3, noting that $[x, y] \in \Gamma^{2} G$, we conclude that $x^{m^{c-1}}=\left(y^{-1} x y\right)^{m^{c-1}}=y^{-1} x^{m c-1} y$, where nil $G=c$. Since $y$ is an arbitrary element of $N$, it follows that $x^{m^{c-1}} \in C_{G}(N)$ and Theorem 2.6 is proved.

REMARK 2.7. Notice that it would have sufficed to assume that $T_{P} \Gamma^{2}\langle x\rangle N$ has finite exponent for all $x \in G$ such that $e x \in C_{G_{Q}}\left(N_{Q}\right)$.

Lorensen's theorem is the key to our relativization of Theorem 1.3, which we now state.

THEOREM 2.8. Let $G$ be a nilpotent group and $N$ a normal subgroup of $G$. Then if $[G, N]$ is a $P$-group of exponent $m, G / C_{G}(N)$ is a P-group of exponent dividing $m^{c-1}$, where nil $G=c$.

Proof. Since $\Gamma_{\langle x\rangle}^{2} N \subseteq[G, N]$, and $[G, N]$ is a $P$-group of exponent $m$, it follows that we have the conditions for applying Lorensen's theorem, so that $e_{0}: C_{G}(N) \rightarrow$ $C_{G_{Q}}\left(N_{Q}\right) Q$-localizes. Now since $[G, N]$ is a $P$-group, its $Q$-localization vanishes, that is, $\left[G_{Q}, N_{Q}\right]=1$. This means that $G_{Q}=C_{G_{Q}}\left(N_{Q}\right)$, so that every $x \in G$ has the property that $e x \in C_{G_{Q}}\left(N_{Q}\right)$. Moreover, $\exp \Gamma_{\langle x\rangle}^{2} N$ divides $m$. Thus, following the proof of Theorem 2.6, we see that $x^{m^{c-1}} \in C_{G}(N)$ for all $x \in G$, so that $\exp \left(G / C_{G}(N)\right) \mid m^{c-1}$. This, of course, implies that $G / C_{G}(N)$ is a $P$-group.

REMARK 2.9. This last implication follows immediately from $G_{Q}=C_{G_{Q}}\left(N_{Q}\right)=C_{G}(N)_{Q}$. 
It remains to provide the relativization of Theorem 1.4. In fact, we may simply relativize each step in Warfield's argument in [4, Corollary 3.16], thus obtaining the following theorem.

THEOREM 2.10. Let $G$ be a nilpotent group and $N$ a normal subgroup such that $G / C_{G}(N)$ is a P-group of exponent $m$. Then $[G, N]$ is a P-group of exponent dividing $m^{c-1}$, where $\operatorname{nil} G=c$.

\section{Appendix}

Homological methods. We show in this appendix how homological arguments may be used to obtain Theorem 1.2, although the numerical estimate is marginally inferior to that given by Theorem 1.4. We emphasize that we have only succeeded in developing a homological method in the absolute case.

We begin with a crucial homological lemma.

LEMMA A.11. Let $G$ be a nilpotent group with nil $G=c$ and let $n \geq 1$. If $G$ is a torsion group with $\exp G=m$, then $m^{n(c-1)+1} H_{n} G=0$.

Proof. We argue by induction on $c$. If $c=1$, then $G$ is commutative. If $K$ is an arbitrary $f g$ subgroup of $G$, then $K$ is a direct product of (finitely many) finite cyclic groups whose orders divide $m$, hence $m H_{n} K=0$. Now $H_{n} G=\lim _{\vec{K}} H_{n} K$, so that $m H_{n} G=0$.

Now we assume $c \geq 2$, and assume the lemma proved for nilpotent groups of class $<c$. We consider the central extension

$$
\Gamma \succ G \longrightarrow G / \Gamma
$$

where $\Gamma=\Gamma^{c} G$, and we exploit the Lyndon-Hochschild-Serre spectral sequence associated with (A.3). In this spectral sequence

$$
E_{p q}^{2}=H_{p}\left(G / \Gamma ; H_{q} \Gamma\right)
$$

Since the universal coefficient formula in homology splits, and since nil $\Gamma=1$, nil $G / \Gamma=$ $c-1$, and $\exp \Gamma|m, \exp (G / \Gamma)| m$, it follows from the inductive hypothesis that, if $p+q>0$,

$$
m E_{p q}^{2}=0, \quad q>0, \quad m^{p(c-2)+1} E_{p 0}^{2}=0 .
$$

(The form of writing in (A.5) and in what follows is acceptable since homology groups and $E_{p q}^{r}$ are additive abelian groups.)

We may then pass to the limit of the spectral sequence, obtaining

$$
m E_{p q}^{\infty}=0, \quad q>0, \quad m^{p(c-2)+1} E_{p 0}^{\infty}=0 .
$$

Now $H_{n} G$ admits a finite filtration

$$
0=F^{-1} \subseteq F^{0} \subseteq \cdots \subseteq F^{p-1} \subseteq F^{p} \subseteq \cdots \subseteq F^{n-1} \subseteq F^{n}=H_{n} G,
$$

such that

$$
F^{p} / F^{p-1}=E_{\infty}^{p q}, \quad p+q=n, \quad 0 \leq p \leq n .
$$


From (A.6) and (A.8) an easy finite induction shows that

$$
m^{p+1} F^{p}=0, \quad 0 \leq p \leq n-1 .
$$

Finally, we exploit the short exact sequence

$$
F^{n-1} \longrightarrow H_{n} G \longrightarrow E_{n 0}^{\infty}
$$

to infer that $m^{n+n(c-2)+1} H_{n} G=0$, or $m^{n(c-1)+1} H_{n} G=0$, completing the inductive step.

Armed with this lemma, we may prove the following theorem.

THEOREM A.12. Let $G$ a nilpotent group with nil $G=c$. Then if $G / Z G$ is a torsion group of exponent $m, G^{\prime}$ is a torsion group of exponent dividing $m^{2 c-2}$.

Proof. We exploit the exact sequence (1.2) and the argument used to prove Schur's theorem. Since nil $G / Z G=c-1$, we know from Lemma A.11 that

$$
m^{2(c-2)+1} H_{2}(G / Z G)=0 .
$$

Thus

$$
m^{2(c-2)+1}\left(G^{\prime} \cap Z G\right)=0 .
$$

Now $G^{\prime} / G^{\prime} \cap Z G \subseteq G / Z G$, so $\exp \left(G^{\prime} / G^{\prime} \cap Z G\right) \mid m$. Putting this together with (A.12), we deduce finally that $G^{\prime}$ is a torsion group and $\exp G^{\prime} \mid m^{2 c-2}$.

We remark (again) that our estimate of $\exp G^{\prime}$ is not best possible.

\section{REFERENCES}

[1] P. Hilton, Localization and cohomology of nilpotent groups, Math. Z. 132 (1973), 263-286. MR 48\#438. Zbl 0264.20037.

[2] P. Hilton and G. Mislin, On the genus of a nilpotent group with finite commutator subgroup, Math. Z. 146 (1976), no. 3, 201-211. MR 53\#615. Zbl 0311.20013.

[3] G. Mislin, Nilpotent groups with finite commutator subgroups, Localization in Group Theory and Homotopy Theory, and Related Topics (Sympos., Battelle Seattle Res. Center, Seattle, Wash., 1974), Lecture Notes in Math., vol. 418, Springer, Berlin, 1974, pp. 103-120. MR 50\#10080. Zbl 0302.20029.

[4] R. B. Warfield Jr., Nilpotent Groups, Lecture Notes in Mathematics, vol. 513, Springer-Verlag, New York, 1976. MR 53\#13413. Zbl 0347.20018.

Peter Hilton: Department of Mathematical Sciences, Suny Binghamton, BinghamTON, NY 13902-6000, USA

Current address: DePARTMENT OF MATHEMATICS, UNIVERSity OF CENTRAL FloridA, ORLANDO, FL 32816-1364, USA

E-mail address: marge@math . binghamton . edu 


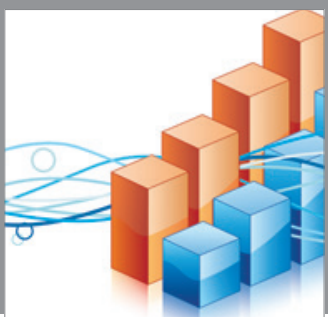

Advances in

Operations Research

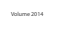

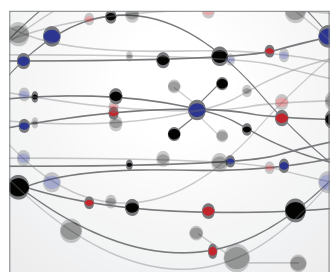

\section{The Scientific} World Journal
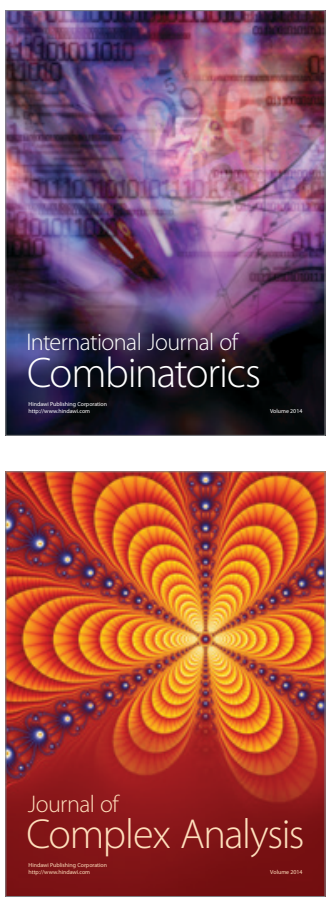

International Journal of

Mathematics and

Mathematical

Sciences
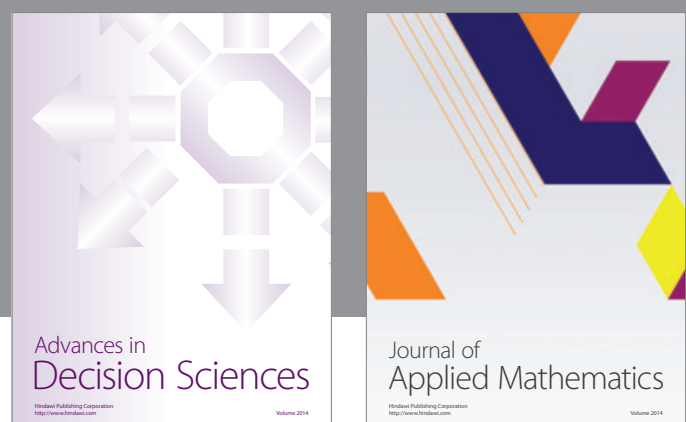

Journal of

Applied Mathematics
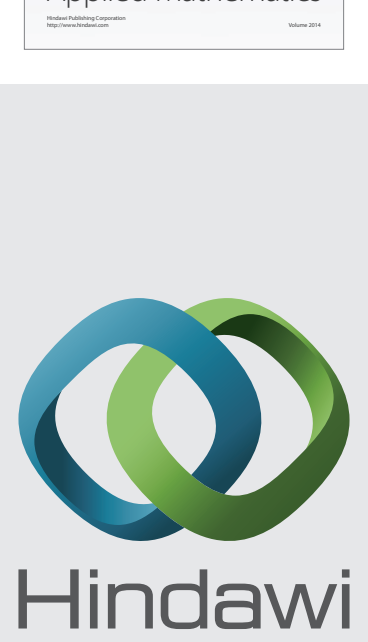

Submit your manuscripts at http://www.hindawi.com
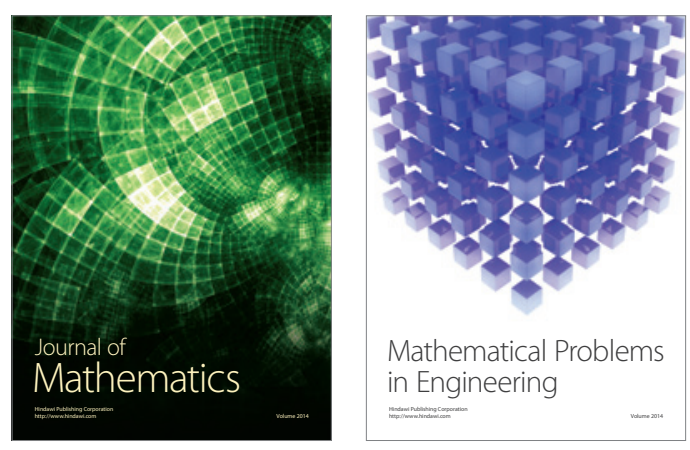

Mathematical Problems in Engineering
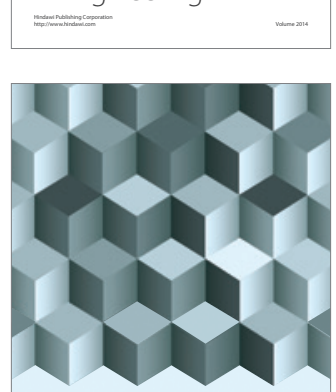

Journal of

Function Spaces
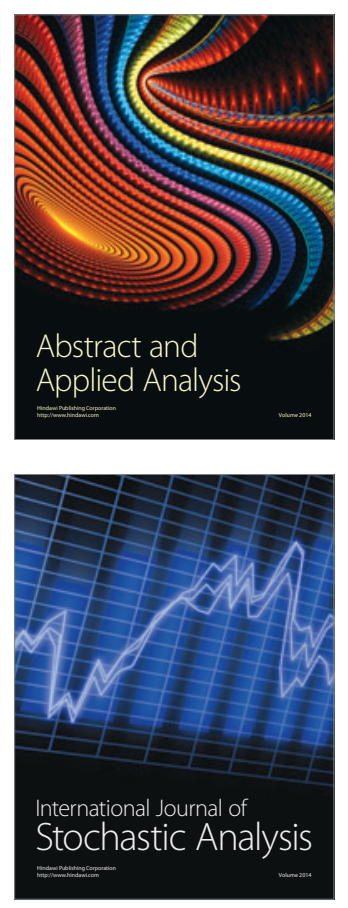

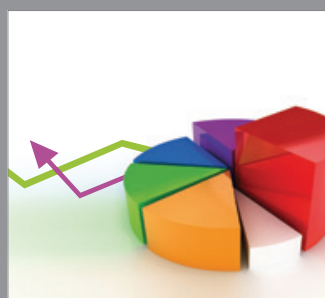

ournal of

Probability and Statistics

Promensencen
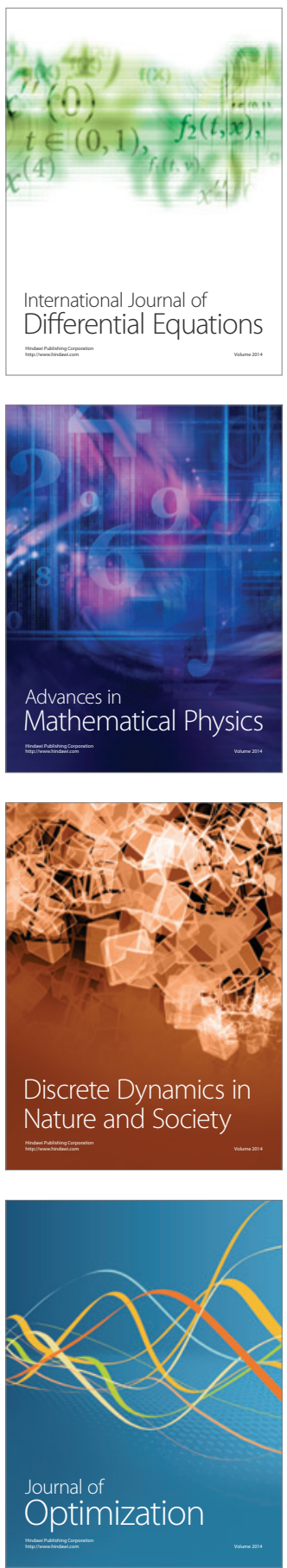